\title{
Il Corpus della scultura dell'alto Medioevo in Italia : struttura e situazione attuale
}

Le corpus de la sculpture du haut Moyen Âge en Italie : structure et situation actuelle

\section{Alessandra Guiglia}

\section{(2) OpenEdition}

\section{Journals}

Edizione digitale

URL: https://journals.openedition.org/cel/19997

DOI: $10.4000 /$ cel. 19997

ISSN: 2262-208X

\section{Editore}

École du Louvre

\section{Notizia bibliografica digitale}

Alessandra Guiglia, " // Corpus della scultura dell'alto Medioevo in Italia : struttura e situazione attuale », Les Cahiers de l'École du Louvre [En ligne], 17 | 2021, mis en ligne le 22 novembre 2021, consulté le 13 décembre 2021. URL : http://journals.openedition.org/cel/19997 ; DOI : https://doi.org/10.4000/cel. 19997

Questo documento è stato generato automaticamente il 13 décembre 2021.

\section{(i) $\ominus$

Les Cahiers de l'École du Louvre sont mis à disposition selon les termes de la licence Creative Commons Attribution - Pas d'Utilisation Commerciale - Pas de Modification 4.0 International. 


\section{Il Corpus della scultura dell'alto Medioevo in Italia : struttura e situazione attuale}

Le corpus de la sculpture du haut Moyen Âge en Italie : structure et situation actuelle

Alessandra Guiglia

Lungo il percorso degli studi sulla scultura altomedievale in Italia, una prima tappa fondamentale è certamente rappresentata dallo straordinario volume di Raffaele Cattaneo L'architettura in Italia dal secolo VI al Mille circa. Ricerche storico-critiche, pubblicato a Venezia nel $1888-89^{1} \mathrm{e}$ in edizione francese nel $1890^{2}$. Nello scorrere le quasi 300 pagine del libro non può sfuggire che il vero interesse del giovane architetto veneto fosse in realtà proprio la scultura, sia architettonica sia liturgica, di quei secoli: basta infatti constatare che delle 170 illustrazioni che accompagnano il testo soltanto una trentina vengono dedicate a piante e alzati di edifici o a particolari architettonici. Le immagini sono in buona parte, 63, tratte dai disegni dell'autore, il quale li realizzò nei suoi numerosi sopralluoghi attraverso la penisola e, significativamente, li siglò con la sua firma in forma di monogramma "all'antica" (fig. 1). Altre 46 sono opera di Giovanni Culluri, collaboratore dell'editore Ongania, che riprodusse per lo più le fotografie scattate dallo stesso Cattaneo; infine 36 (contrassegnate da un asterisco) sono tratte da opere di altri autori, tra le quali rivestono particolare interesse quelle che riproducono le sculture di vari siti della Siria bizantina ${ }^{3}$. 
Fig. 1. « Particolari del protiro di Cimitile - principio del sec. VIII »

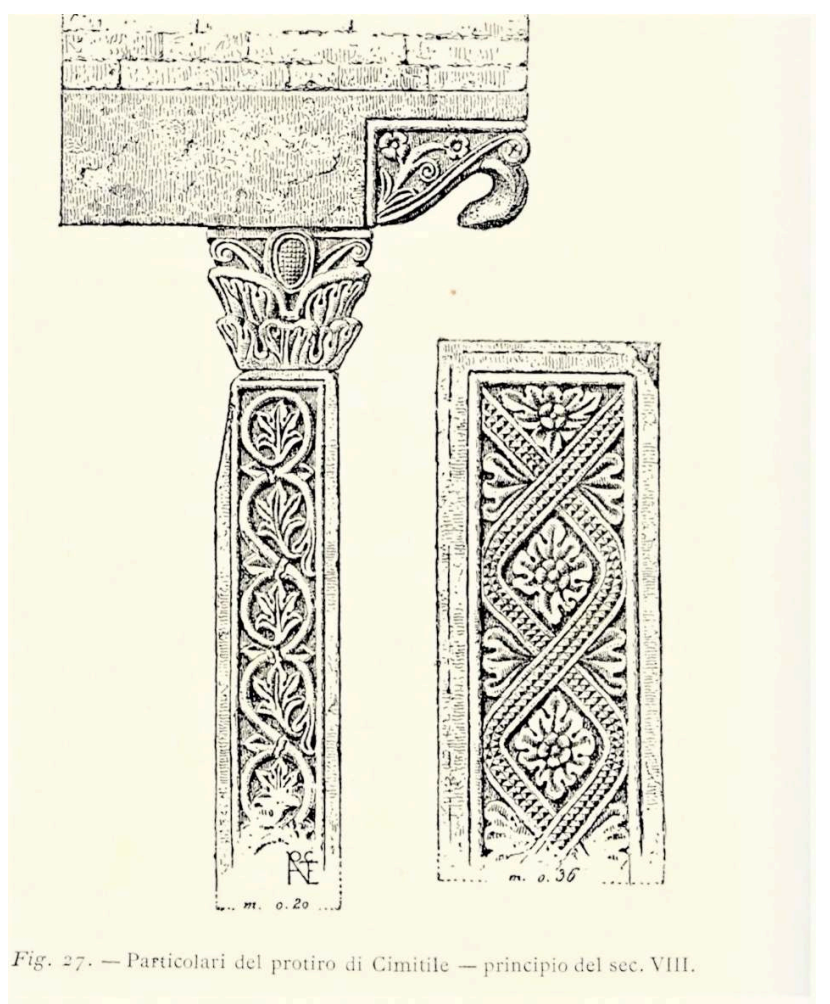

Da R. Cattaneo, 1888-89, fig. 27.

2 Le molte brillanti intuizioni e le acute osservazioni sulle sculture, anche in merito alla cronologia, risultano in buona parte ancor oggi valide, così come meritorie furono l'apertura dell'orizzonte critico verso l'Oriente bizantino e la consapevolezza dell'impatto della cultura greca sulla tradizione romano-occidentale allora prevalente negli studi ${ }^{4}$. La morte repentina per febbri da vaiolo a soli 28 anni, il 6 dicembre 1889, gli ha impedito di continuare a coltivare la sua vera passione per lo studio della scultura e ha privato noi del ricchissimo archivio di foto e disegni, che fu allora bruciato per evitare i pericoli del contagio ${ }^{5}$. Resta la grandezza della sua opera scritta, che costituisce un imprescindibile punto di riferimento per chiunque voglia affrontare lo studio dell'architettura e della scultura dei secoli dell'alto medioevo ${ }^{6}$.

Di lì a pochi anni, nel 1896, appare un altro scritto di fondamentale interesse, anche dal punto di vista metodologico, pur se circoscritto quasi esclusivamente a Roma e all'ambito laziale. Si tratta de «La scultura ornamentale romana nei bassi tempi» di Ferdinando Mazzanti, pubblicato nella prestigiosa rivista di Adolfo Venturi Archivio Storico dell'Arte in due fascicoli successivi illustrati con i disegni dell'autore ${ }^{7}$. Architetto anch'egli, Mazzanti coltivò una passione davvero straordinaria per la scultura altomedievale che lo condusse oltre la semplice illustrazione dei marmi e lo spinse alla ricerca spasmodica anche del più piccolo frammento in ogni angolo della città e del territorio laziale per offrirne la documentazione più completa (fig. 2), sia con il disegno sia anche con l'uso dello "spolvero" o "frottage" e proponendo ricostruzioni talora attendibili talora del tutto fantasiose. Diversamente dallo sfortunato destino dei materiali di Cattaneo, restano di Mazzanti migliaia di disegni delle sculture romane che ci fanno comprendere perfettamente il suo metodo di lavoro e di studio ${ }^{8}$. Ma su questo torneremo in dettaglio più avanti. 
Fig. 2. «Pluteo del sec. VIII in S. Cosimato»

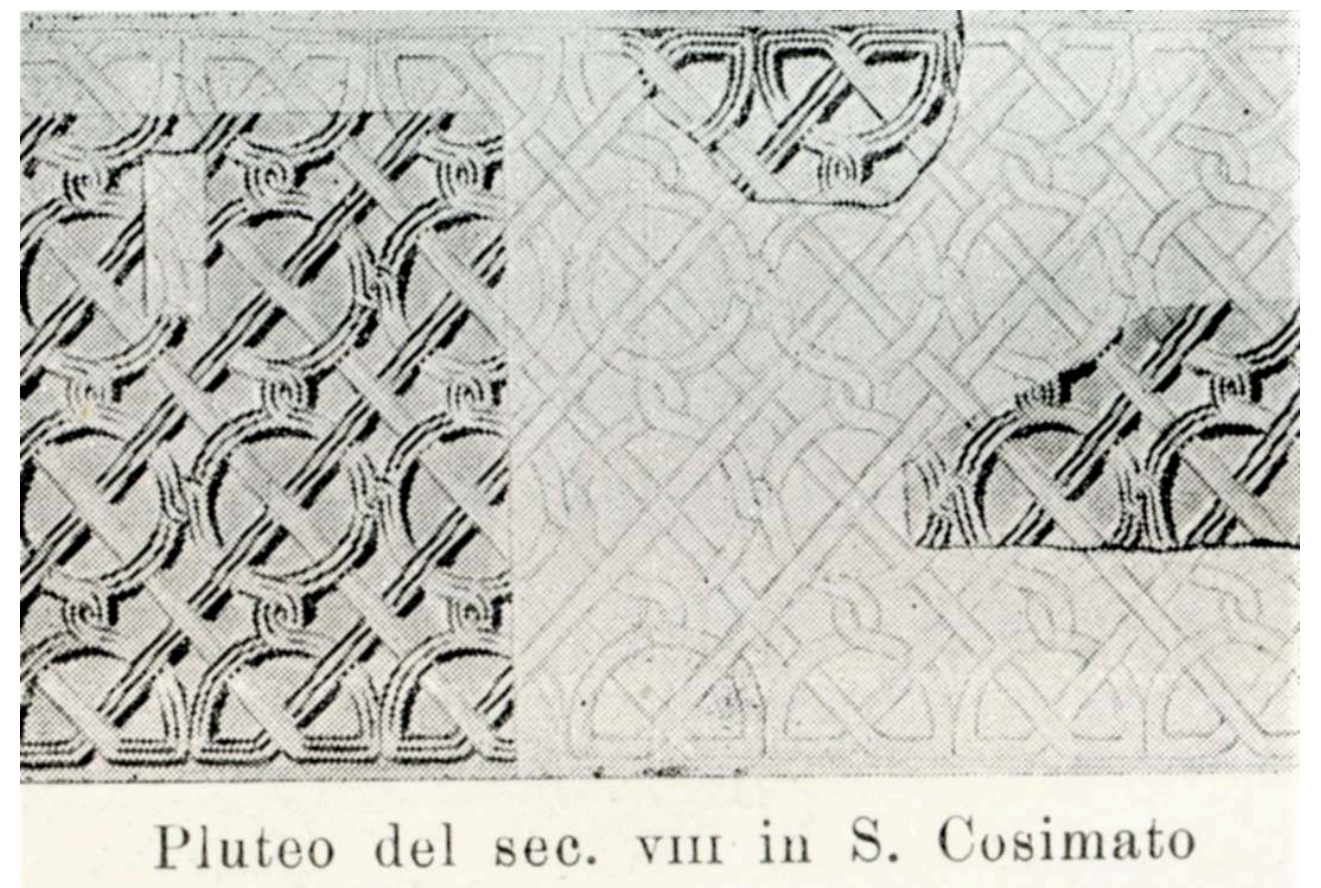

Da F. Mazzanti, 1896, p. 162.

Diverso tra i due studiosi era anche l'approccio critico sulle origini della scultura dell'alto medioevo, pur se con la comune premessa che qualificava come "barbari" quei secoli: decisamente innovativo, e in qualche modo audace, Cattaneo, nel sostenere il ruolo determinante della componente bizantina; più tradizionalista Mazzanti, nella ferma convinzione che vi fosse una continuità ininterrotta dall'età romana al XII secolo e all'arte dei Cosmati. In conclusione, due prospettive differenti ma che hanno posto al centro delle loro ricerche in primo luogo il manufatto scolpito, l'analisi autoptica e l'accurata riproduzione ${ }^{9}$.

Nei decenni successivi, fino ai primi anni Cinquanta del Novecento il dibattito critico si concentrò proprio sul problema delle origini di quella che allora veniva definita "scultura cosiddetta longobarda", per la quale furono chiamate in causa le culture artistiche più diverse, dall'Oriente all'arte romana, appunto, ma anche dall'area germanica, celtica e irlandese ${ }^{10}$. Tra le figure emergenti sullo sfondo di questo dibattito ricordo innanzitutto Rudolf Kautzsch ${ }^{11}$, Arthur Haseloff ${ }^{12}$, Paolo Verzone ${ }^{13}$, Carlo Cecchelli ${ }^{14}$, e Géza de Francovich ${ }^{15}$, solo per menzionare coloro che più di altri, in quel torno di tempo, si sono concentrati, con diversi accenti, sulle testimonianze del territorio italiano.

6 Cruciale per i nostri studi è stato il $1^{\circ}$ Congresso Internazionale di Studi Longobardi, tenutosi a Spoleto nel 1951: il 30 settembre, su istanza di Mario Salmi, Carlo Cecchelli, Gianpiero Bognetti, Gaetano Panazza ed altri, il Congresso «constatata l'urgenza e la necessità di una maggiore tutela, da parte degli organi competenti, del materiale artistico ed epigrafico dell'età alto medioevale, onde impedirne la deprecata distruzione che tuttora si verifica in molti casi; ritenendo essere indispensabile la raccolta di tutti i monumenti di quei secoli con unità di vedute e con serietà per un più 
approfondito studio dell'arte alto medioevale; fa voti ... che venga concretata l'iniziativa di raccogliere e pubblicare un corpus dei monumenti preromanici (architettura, pittura, scultura, arti applicate) ${ }^{16}$. Il Congresso, inoltre, fece voto che a Spoleto stessa fosse istituito un Centro di studi sull'alto medioevo. Cosa che in effetti avvenne l'anno seguente, il 7 giugno 1952, per iniziativa di Giuseppe Ermini, allora Rettore dell'Università di Perugia poi Ministro della Pubblica Istruzione. Tra le prime iniziative del CISAM ci fu proprio quel Corpus auspicato in sede di Congresso, ed in particolare quello dedicato alle sculture, giudicato il più urgente da Mario Salmi «essendo [le sculture] più suscettibili di dispersione e distruzione» ${ }^{17}$. Lo stesso Salmi prese in cura la pubblicazione dei volumi firmandone in quasi tutti la Premessa fino alla fine degli anni Settanta ${ }^{18}$.

7 Gli anni immediatamente successivi alla metà del Novecento sono dunque stati di grande importanza per lo studio della scultura altomedievale in Italia. Agli eventi ora ricordati ne va senz'altro aggiunto un altro, particolarmente significativo per la città di Roma, cioè l'istituzione di un Museo dell'Alto Medioevo, voluta ancora una volta da Mario Salmi e deliberata dal Consiglio Superiore di Antichità e Belle Arti il 22 febbraio $1955^{19}$.

8 Veniamo ora con maggiore dettaglio al Corpus. Il coordinamento dei volumi è affidato ad un Comitato scientifico della Fondazione CISAM che, fino al settembre 2018, era composto da Letizia Pani Ermini, vera figura strategica dell'impresa editoriale negli ultimi decenni, insieme ad Adriano Peroni, Silvana Casartelli Novelli e, più recentemente, Claudia Barsanti (fino alla sua scomparsa nel 2017), chi scrive, ed infine Manuela Gianandrea. In seguito alla morte di Letizia Pani Ermini, avvenuta il 26 settembre 2018, il Comitato è stato a lungo in fase di ricomposizione, a causa anche delle difficoltà legate alla pandemia, fino a che il 5 gennaio 2021 si è riunito per la prima volta con nuovi e vecchi componenti: nell'ordine Fabrizio Crivello, Francesca Romana Stasolla, e ancora Silvana Casartelli Novelli, Manuela Gianandrea e chi scrive ${ }^{20}$.

Con l'aiuto di una cartina (fig. 3) e di un elenco in ordine cronologico dei volumi già pubblicati è possibile avere un'idea del procedere del Corpus sia sul territorio sia nel tempo. 
Fig. 3. Carta della penisola italiana con indicazione delle diocesi già pubblicate

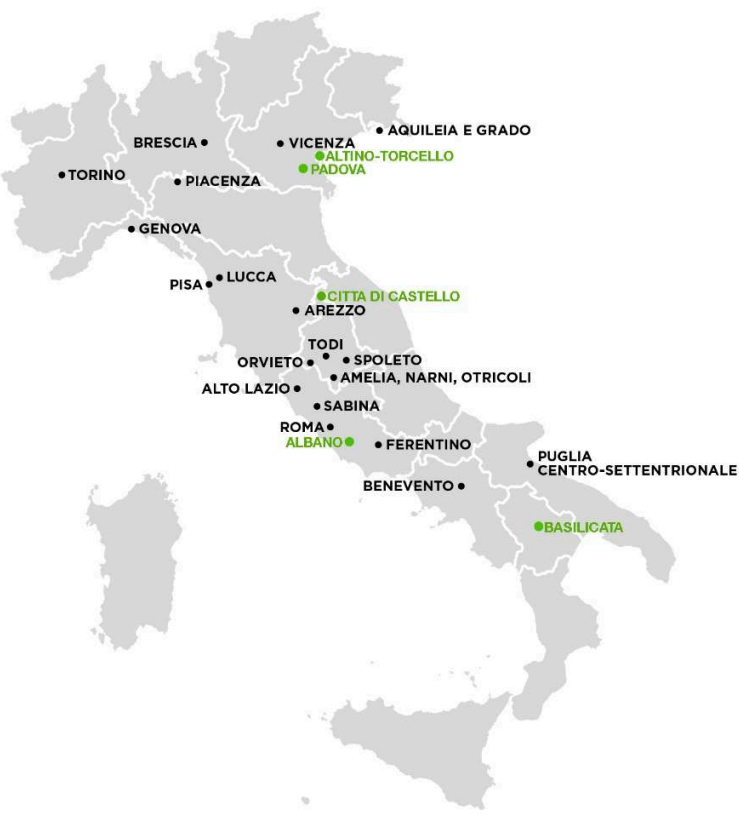

Carta della penisola italiana con indicazione delle diocesi già pubblicate (in nero) e di quelle in corso di pubblicazione (in verde). Elaborazione grafica Studio Azimut, Roma.

I - La diocesi di Lucca, 1959, Isa Belli Barsali

II - La diocesi di Spoleto, 1961, Joselita Serra

III - La diocesi di Brescia, 1966, Gaetano Panazza, Amelio Tagliaferri

IV - La diocesi di Genova, 1966, Colette Dufour Bozzo

V - La diocesi di Benevento, 1966, Mario Rotili

VI - La diocesi di Torino, 1974, Silvana Casartelli Novelli

VII, 1- La diocesi di Roma. La IV regione ecclesiastica, 1974, Letizia Pani Ermini

VII, 2 - La diocesi di Roma. Le raccolte dei Fori Imperiali, 1974, Letizia Pani Ermini

VII, 3 - La diocesi di Roma. La II regione ecclesiastica, 1974, Alessandra Melucco Vaccaro

VIII - Le diocesi dell'Alto Lazio (Bagnoregio, Bomarzo, Castro, Civita Castellana, Nepi, Orte, Sutri, Tuscania), 1974, Joselita Raspi Serra

VII, 4 - La diocesi di Roma. La I regione ecclesiastica, 1976, Margherita Trinci Cecchelli

IX - La diocesi di Arezzo, 1977, Alberto Fatucchi

X - Le diocesi di Aquileia e Grado, 1981, Amelio Tagliaferri

VII, 5 - La diocesi di Roma. Il Suburbio. I, 1981, Umberto Broccoli

XI - La diocesi di Ferentino, 1983, Anna Maria Ramieri

XII - Le diocesi di Amelia, Narni, Otricoli, 1985, Gioia Bertelli

XIII - La diocesi di Todi, 1993, Francesca d'Ettorre 
VII, 6 - La diocesi di Roma. Il Museo dell'Alto Medioevo, 1995, Alessandra Melucco Vaccaro, Lidia Paroli

XIV - La diocesi di Vicenza, 2001, Ettore Napione

XV - Le diocesi della Puglia centro-settentrionale (Aecae, Bari, Bovino, Canosa, Egnathia, Herdonia, Lucera, Siponto, Trani, Vieste), 2002, Gioia Bertelli

XVI - La diocesi di Orvieto, 2003, Donatella Scortecci

XVII - La diocesi di Sabina, 2005, Fabio Betti

XVIII - La diocesi di Piacenza e il monastero di Bobbio, 2008, Eleonora Destefanis

XIX - La diocesi di Pisa, 2011, Laura Testi Cristiani

VII, 7 - La diocesi di Roma. La III regione ecclesiastica, 2015, Claudia Barsanti, Roberta Flaminio, Alessandra Guiglia

Il primo volume, dedicato alla diocesi di Lucca, ha visto la luce nel 1959 e l'ultimo, il venticinquesimo fino ad oggi e il settimo relativo alla diocesi di Roma, nel 2015, con una distribuzione nel tempo abbastanza cadenzata, che vede tuttavia due picchi nel 1966 e nel 1974 (con i primi tre volumi della diocesi di Roma e quello dell'Alto Lazio). Nonostante questo arco cronologico piuttosto ampio - 56 anni - l'impostazione di base non è sostanzialmente mutata. L'analisi del territorio segue l'articolazione delle diocesi ecclesiastiche, una soluzione di comodo che deve comunque prevedere una certa flessibilità, soprattutto a riguardo dei confini che possono essere mutati nel tempo ed essere, dunque, talora di difficile definizione per i secoli dell'alto medioevo. Nel caso specifico di Roma la suddivisione interna segue l'estensione delle regioni ecclesiastiche, che solo in parte coincidono con quelle augustee e si prestano anche in questo caso a qualche difficoltà nell'identificazione dei confini.

36 Anche la struttura del testo non ha subito cambiamenti rilevanti. In primo luogo un'introduzione storico-topografica alla diocesi (o alla regione ecclesiastica nel caso di Roma), quindi un panorama complessivo diacronico sulla consistenza delle sculture, infine il catalogo dei materiali preceduto da una breve introduzione all'edificio cui essi sono pertinenti.

7 Nel catalogo vengono per lo più rispettate delle regole di massima che prevedono, per ciascun sito, un ordine cronologico dei pezzi e all'interno di esso un ordine tipologicofunzionale e dimensionale. In alcuni casi più complessi, come ad esempio l'insieme dei materiali della chiesa di San Clemente a Roma (170 schede), si è proceduto eccezionalmente in ordine topografico ${ }^{21}$.

Nel corso di quasi sessant'anni ci sono stati comunque, come è ovvio, alcuni importanti mutamenti. Innanzitutto una maggiore flessibilità dei limiti cronologici che sono, da un lato, arretrati fino al IV-V secolo rispetto al VI secolo iniziale e, dall'altro, si sono estesi al pieno XI secolo, soprattutto in relazione ai contesti archeologici e architettonici di pertinenza ma anche per una documentazione il più possibile completa ${ }^{22}$. Particolare attenzione viene ora rivolta proprio a questi due estremi cronologici, intesi come periodi di transizione, e soprattutto al secondo che individua nel passaggio tra X e XI secolo una fase sensibile nella formazione della scultura del pieno medioevo.

Negli ultimi decenni, grazie anche all'implemento del materiale di confronto pubblicato via via nei Corpora, è stato possibile offrire un migliore inquadramento a livello nazionale ed evidenziare la diffusione di temi e schemi decorativi, anche in relazione alle consuetudini di bottega e al problema delle maestranze itineranti. Occupano 
inoltre uno spazio maggiore le osservazioni di carattere tecnico sui metodi di lavorazione e sono sempre più presenti le ricostruzioni grafiche e le ricomposizioni fotografiche dei materiali frammentari.

Va infine ricordata una piccola ma significativa innovazione di carattere formale: fino al volume del 2002 sulle diocesi della Puglia centro-settentrionale la copertina era rimasta molto semplice e sobria, mentre a partire dal volume del 2003 sulla diocesi di Orvieto essa acquista una veste più vivace e graficamente consona ai contenuti con fantasiosi motivi a intreccio. Da allora i volumi vengono editi dalla nuova Fondazione CISAM e la collana è stata diretta da Letizia Pani Ermini e da Adriano Peroni. Tornando ora alla carta della penisola, possiamo facilmente constatare che i venticinque volumi pubblicati coprono il territorio in maniera non sistematica né omogenea. Al nord sei regioni (Piemonte, Liguria, Lombardia, Emilia Romagna, Veneto e Friuli-Venezia Giulia) sono rappresentate da almeno una diocesi, al sud solo due regioni (Campania e Puglia), ma con dodici diocesi, mentre al centro in tre regioni (Toscana, Umbria e Lazio) si contano ben venti diocesi (di cui otto raggruppate nel volume dell'Alto Lazio). In conclusione un campione ancora non particolarmente nutrito, costituito da undici regioni e trentotto diocesi.

Nonostante ciò, le circa seimila sculture finora censite e analizzate offrono già un quadro sufficientemente chiaro della ricchezza e della varietà dei materiali, nonché delle peculiarità compositive, stilistiche e di repertorio che contraddistinguono le diverse aree storico-culturali e artistiche del territorio italiano nel corso dei secoli dell'alto medioevo, pur essendo evidente tra VIII e IX secolo l'utilizzo generalizzato e ubiquitario, nel lessico decorativo, dell'intreccio vimineo nelle più diverse declinazioni,

Poiché la struttura editoriale di questo contributo non consente quell'excursus per immagini che ha accompagnato la presentazione dello scorso anno, mi limiterò a richiamare solo alcuni casi esemplificativi a partire dalla diocesi di Torino, con le originalissime sculture provenienti dal complesso del S. Salvatore, di datazione discussa tra l'età del vescovo Claudio l'iconoclasta (818-827) e la fine del IX secolo ${ }^{23}$, alla diocesi di Genova, per la quale ricordo soprattutto i singolari capitelli da S. Tommaso al Museo di S. Agostino, attribuiti agli anni di poco precedenti al $1000^{24}$, ma anche altre sculture che per molti versi si riallacciano a modelli bizantini dell'età dei Macedoni come la lastra con pavoni e il sarcofago di Santa Marta ${ }^{25}$; infine alla diocesi di Piacenza con il celebre complesso di rilievi del monastero di Bobbio, tra i quali spicca la lastra di Cumiano del tempo di Liutprando, riscolpita in età carolingia ${ }^{26}$. Procedendo verso est, la diocesi di Brescia ci porta nel cuore della scultura longobarda con la collezione del Museo Cristiano, oggi Santa Giulia Museo della Città, ben rappresentata dalla splendida lastra con pavone, assegnata ormai alla prima metà dell'VIII secolo ${ }^{27}$; i materiali della diocesi di Vicenza, forse meno noti, hanno offerto l'opportunità di individuare un'officina lapidaria, detta berico-benacense, attiva nella prima età carolingia nell'area tra la sponda orientale del lago di Garda e la stessa Vicenza ${ }^{28}$. Più articolata è evidentemente la situazione delle diocesi di Aquileia e Grado, aperte verso l'Oriente bizantino $^{29}$ : da un lato le sculture del VI secolo delle chiese di Grado, tardo riflesso locale della produzione dell'età di Giustiniano ${ }^{30}$, cui si affiancano le testimonianze di manifattura costantinopolitana, come il capitello imposta a traforo con cornucopie in S. Maria delle Grazie ${ }^{31}$; dall'altro lato le celebri opere della committenza longobarda di Cividale, come ad esempio l'altare di Ratchis o il complesso di sculture del tegurio del «battistero di Callisto» ${ }^{32}$. 

Toscana: Lucca, Arezzo, Pisa, e mostrano caratteri piuttosto disomogenei ${ }^{33}$ con molti materiali databili sullo scorcio del X secolo, come i capitelli di Arezzo e Cortona ${ }^{34}$ o ancora alcuni di quelli della celebre abbazia di S. Antimo, di sapore ormai romanico ${ }^{35}$; in diocesi di Pisa, oltre alla diffusione di una scultura architettonica databile già all'XI secolo inoltrato, si rileva un'incisiva presenza di temi figurati, sia di VIII sia di X-XI secolo, come testimoniano le due lastre di Lupeta ${ }^{36}$. Sei diocesi sono in Umbria: Spoleto, Todi, Orvieto, quindi Amelia, Narni, Otricoli, queste ultime tre riunite in un solo volume. Tra le opere più significative ricordo la lastra di S. Pietro in Valle a Ferentillo, opera di Ursus Magester degli anni $739-742^{37}$, il meno noto ciborio di S. Cristina a Bolsena, dell'inizio dell'VIII secolo ${ }^{38}$, e il caso piuttosto raro di altare integro in S. Martino a Taizzano della fine dello stesso secolo ${ }^{39}$; merita attenzione anche la bella lastra con agnelli ai lati di una croce gemmata nel duomo di Narni, della metà del VI secolo ${ }^{40}$. Delle undici diocesi del Lazio, otto sono riunite in uno stesso volume, quello dell'Alto Lazio: prevalgono i temi decorativi ad intreccio vimineo e a girali, spesso a rete di maglie di quadrati e rettangoli con variatissimi inserti vegetali e animali ${ }^{41}$, ma sono testimoniate anche scene figurate di notevole impegno come la caccia sul sarcofago del duomo di Civita Castellana, datata epigraficamente al IX secolo ${ }^{42}$. Ricordo inoltre un arredo liturgico di indiscussa importanza come la cosiddetta "iconostasi" di S. Leone a Capena, che, benché rimaneggiata all'inizio del XVI secolo, mostra ancora parti essenziali della struttura del tempo di papa Leone IV (metà del IX secolo), tra le quali l'epistilio con archetto centrale ${ }^{43}$. Segnalo, infine, per la diocesi di Sabina, l'eccezionale serie di capitelli a stampella dell'abbazia di Farfa, collocabile nei decenni centrali dell'VIII secolo, probabilmente in coincidenza con la presenza di abati provenienti dall'Aquitania ${ }^{44}$.

Le diocesi del sud, Benevento in Campania e ben dieci nella Puglia centrosettentrionale, compresa Bari, riunite in un solo volume, rivelano una concreta apertura verso le correnti stilistiche della riva opposta dell'Adriatico e ancor più lontano verso la capitale bizantina. Lo dimostrano chiaramente sia i capitelli del S. Nicola di Bari con trofei di cornucopie, di sicura importazione costantinopolitana ${ }^{45}$, sia le lastre a pseudo transenna della basilica di S. Michele Arcangelo a Monte Sant'Angelo ${ }^{46}$, insieme a quelle analoghe del Museo del Sannio di Benevento ${ }^{47}$, forse provenienti da Siponto, che sono state ormai tutte ricondotte unanimemente ai primi decenni del VI secolo.

Molto è stato fatto, ma molto di più resta ancora da fare, pur con la consapevolezza che l'intensificarsi sempre maggiore degli studi sulla scultura altomedievale, da un lato, e il succedersi di scavi e indagini sui monumenti di quel tempo, dall'altro, rendono ormai urgente una riflessione sull'opportunità di un eventuale aggiornamento dei volumi più datati, cui, peraltro, già da qualche anno, Letizia Pani Ermini stava pensando.

Intanto la Fondazione CISAM procede con la pubblicazione di nuovi volumi, come è evidenziato in verde nella cartina (fig. 3): le diocesi di Padova, Altino-Torcello, Città di Castello, Albano, e quelle della Basilicata sono infatti pronte per la stampa; altre diocesi del territorio ligure, piemontese, lombardo, laziale e campano sono già in lavorazione.

Abbiamo finora tralasciato la situazione di Roma, che si presenta, per la complessità del territorio urbano e suburbano assai articolata. Sono apparsi finora sette volumi, che comprendono le prime quattro regioni ecclesiastiche ${ }^{48}$, evidenziate nella carta della città (fig. 4); il Suburbio settentrionale, con i complessi monumentali di S. Lorenzo e S. 
Agnese fuori le mura e con numerosi cimiteri posti lungo le vie Salaria, Nomentana e Tiburtina ${ }^{49}$; infine due importanti collezioni museali: il Museo dell'Alto Medioevo ${ }^{50} \mathrm{e} \mathrm{la}$ Raccolta dei Fori Imperiali ${ }^{51}$.

Fig. 4. Carta topografica della diocesi di Roma con indicazione delle regioni finora pubblicate.

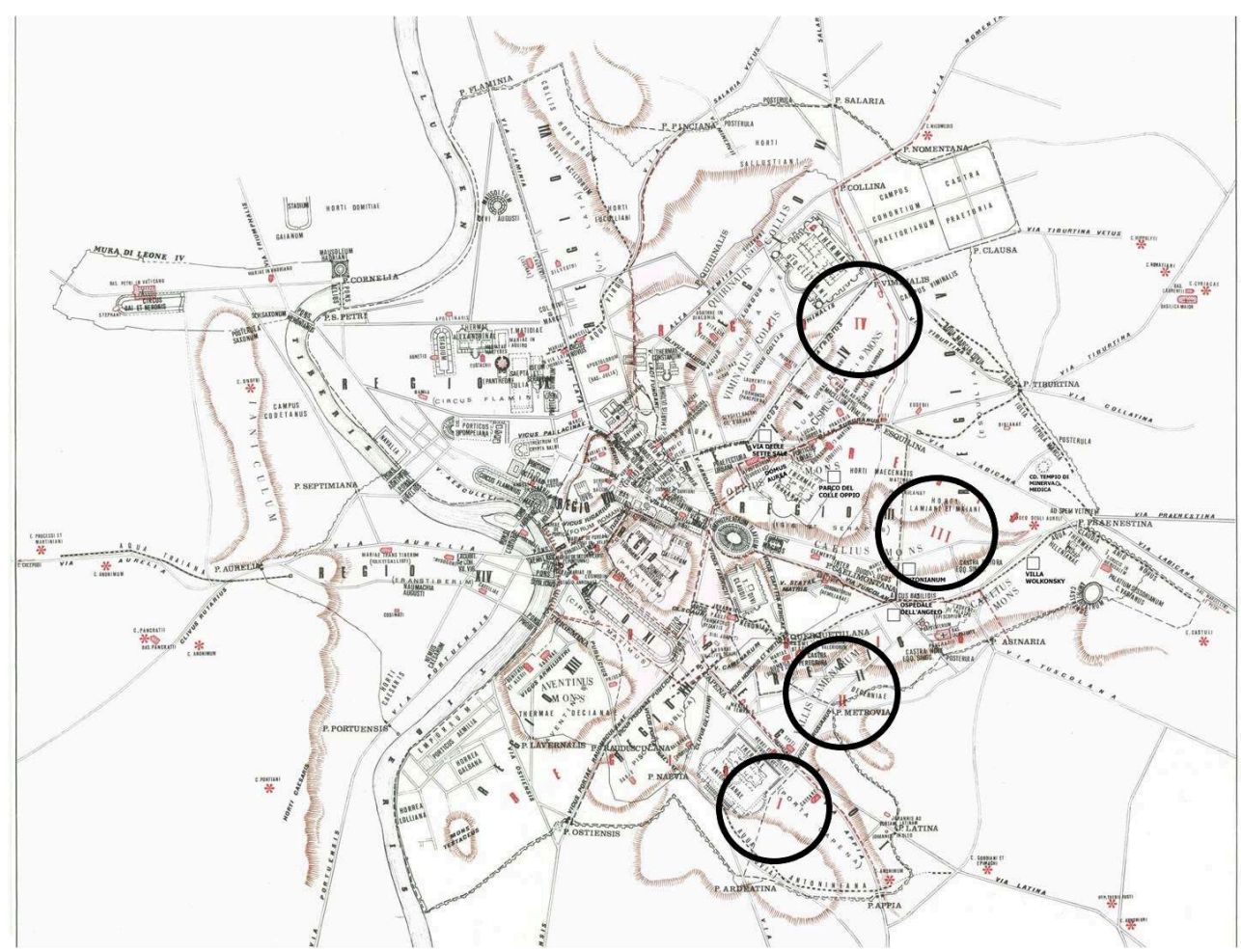

Carta topografica della diocesi di Roma con indicazione delle regioni finora pubblicate. Rielaborazione grafica da C. Barsanti, R. Flaminio, A. Guiglia, 2015.

E' ormai quasi pronto per la stampa il volume dedicato alla VII regione, il Trastevere, mentre sono ancora in preparazione quelli delle regioni V e VI (l'area urbana centrale), dell'area del Foro Romano e del Palatino e infine quello del Suburbio meridionale con il Vaticano.

Al piano generale dell'opera dedicata a Roma, iniziata nel 1974, si auspica che si possa aggiungere ora un nuovo volume, fortemente voluto negli ultimi anni da Letizia Pani Ermini, che renderà noti i materiali altomedievali - circa quattrocento pezzi conservati nel Museo di Roma a Palazzo Braschi. Di essi solo una minima parte è pubblicata $^{52}$ e oggi esposta nelle nuove sale del Museo, mentre gli altri, collocati nel Lapidario, sono in corso di studio e catalogazione. Essi provengono in gran parte da scavi urbani della seconda metà dell'ottocento e dai cantieri delle demolizioni della prima metà del Novecento, soprattutto nell'area fra il Teatro di Marcello e la chiesa di S. Maria in Cosmedin, dove sorgeva un fitto tessuto abitativo. Ciò che giustificherebbe la presenza di un cospicuo gruppo di architravi e stipiti di porte e finestre, anche di ridotte dimensioni (fig. 5). 
Fig. 5. Museo di Roma a Palazzo Braschi

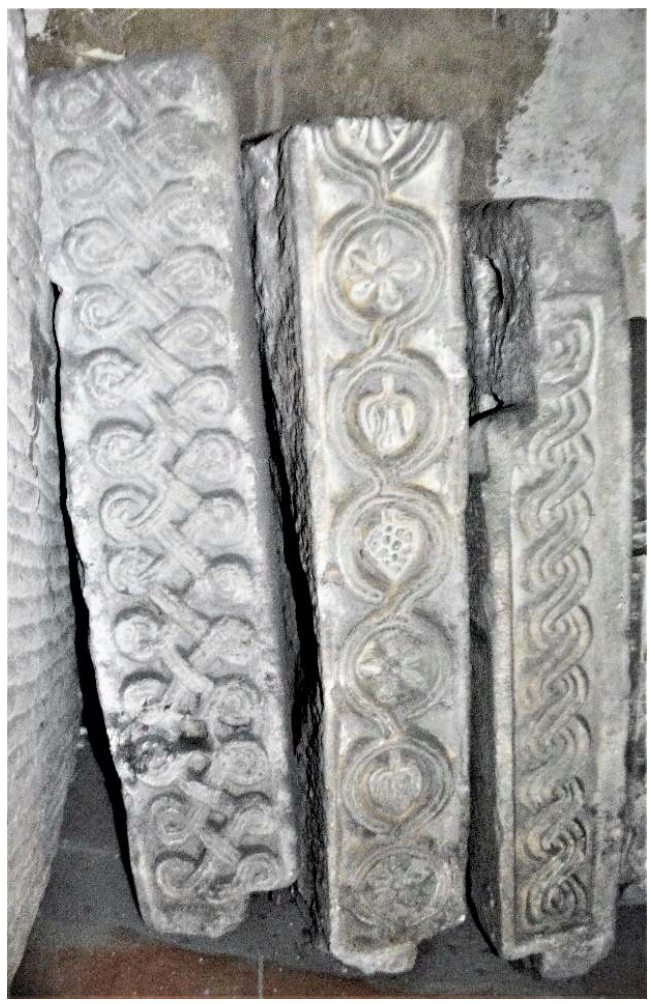

Lapidario: stipiti o architravi con decorazione ad intrecci viminei.

(c) A. Guiglia

51 Di estrema importanza è infine il progetto di pubblicazione dei circa tremila disegni di Ferdinando Mazzanti, dei quali ho fatto cenno all'inizio. Un progetto in gestazione già da molti anni, perseguito con tenacia da Silvana Casartelli Novelli e Letizia Pani Ermini, che si sta ora finalmente concretizzando con l'uscita del primo volume, edito anch'esso dalla Fondazione CISAM $^{53}$. I disegni sono oggi conservati presso la Galleria Nazionale d'Arte Moderna che li acquistò nel 1903, qualche anno dopo la morte dell'architetto ${ }^{54}$. Un primo nucleo di oltre cinquecento disegni relativi al territorio laziale è stato pubblicato nel $2002^{55}$, ma la parte più cospicua, quella della città di Roma, è ancora quasi del tutto inedita, se non per casi specifici riferiti a singoli monumenti, come ad esempio le sculture della recinzione liturgica di S. Clemente del VI secolo e altri marmi analoghi $^{56}$ o quelle della basilica di S. Sabina all'Aventino, pertinenti al recinto presbiteriale del IX secolo ${ }^{57}$, ma anche a singoli disegni di sculture ${ }^{58}$. Il ricchissimo materiale è composto soprattutto da disegni, schizzi, appunti e misure, tracciati a penna o a matita su supporti di ogni tipo (fig. 6), persino biglietti del tram, ma anche da tavole acquerellate, "spolveri" o calchi di notevoli dimensioni (fig. 7) e ricostruzioni grafiche. In molti casi i disegni e gli schizzi di Mazzanti costituiscono oggi l'unica testimonianza di sculture ormai scomparse, mentre in altri casi costituiscono invece preziosi indizi per il ritrovamento di materiali tuttora esistenti ma non ancora individuati. 
Fig. 6. « S. Maria in Trastevere nel cortiletto della sacrestia »

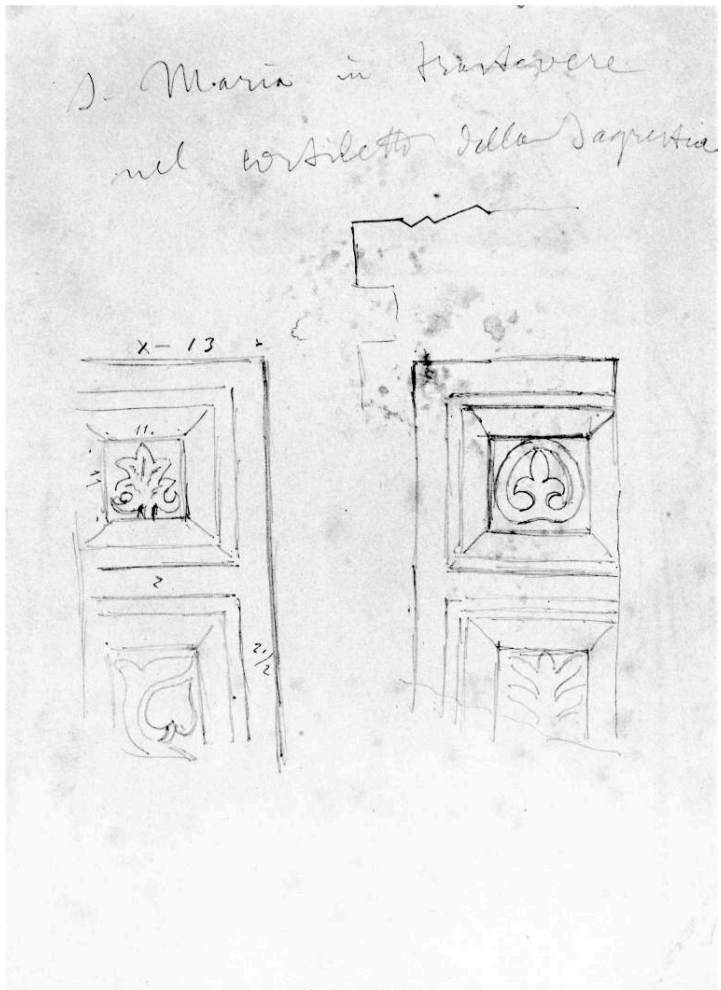

Roma, Galleria Nazionale di Arte Moderna, Fondo Mazzanti, disegno a matita su carta delle due facce opposte di un frammento di pilastrino: «S. Maria in Trastevere nel cortiletto della sacrestia » (da C. Barsanti, R. Flaminio, A. Guiglia, 2015). 
Fig. 7. "spolvero" di un pilastrino della schola cantorum

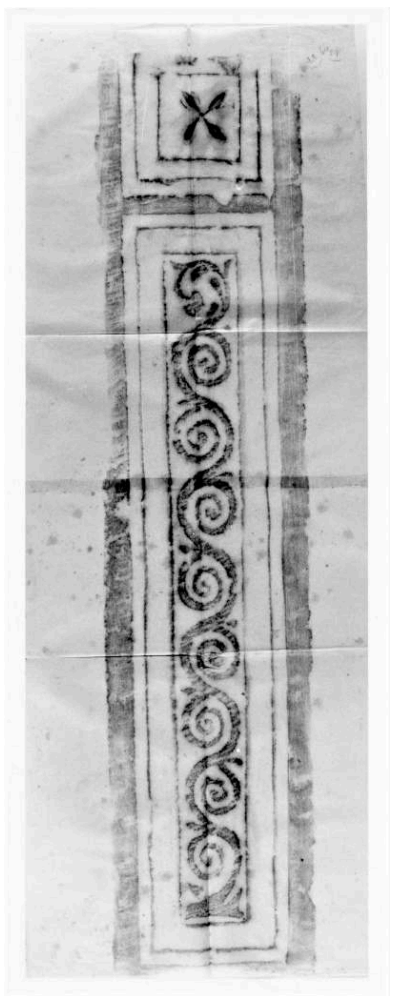

Roma, Galleria Nazionale di Arte Moderna, Fondo Mazzanti, "spolvero" di un pilastrino della schola cantorum di S. Clemente a Roma (da C. Barsanti, R. Flaminio, A. Guiglia, 2015).

Il progetto prevede la pubblicazione dei materiali grafici secondo l'ordine delle regioni ecclesiastiche, in modo da affiancare coerentemente i nuovi volumi a quelli del corpus delle sculture ed evidenziare l'eccezionale valore documentario dei disegni, in rapporto all'esistente. Il primo volume, curato da Manuela Gianandrea e dedicato appunto alla I regione ecclesiastica, contiene un'ampia introduzione su Mazzanti e i disegni relativi al territorio dell'Aventino, con le sue numerose chiese, tra le quali principalmente $\mathrm{S}$. Sabina, e alle aree delle Terme di Caracalla e della Marmorata.

Ci auguriamo che presto possano essere avviati anche i volumi successivi, così da rendere pienamente fruibile un patrimonio preziosissimo non solo per la conoscenza della scultura di Roma ma anche per lo studio più ampio della scultura dell'alto medioevo.

\section{NOTE}

1. Raffaele Cattaneo, L'architettura in Italia dal secolo VI al Mille circa. Ricerche storico-critiche, Venezia, Ferdinando Ongania Editore, 1888 (ma a p. 294 viene specificato che il volume fu finito di stampare il 20 luglio 1889). 
2. L'architecture en Italie $\mathrm{du} \mathrm{VI}^{e}$ au $\mathrm{XI}^{e}$ siècle. Recherches historiques et critiques, Venise, Ferdinando Ongania Editore, 1890.

3. In questo caso Cattaneo si avvalse dell'opera di Melchior de Vogüé, Syrie Centrale. Architecture civile et religieuse du $I^{e r}$ au VII ${ }^{e}$ siècle, Paris, 1865-1877, studioso per il quale egli nutriva particolare ammirazione. Si veda ad esempio R. Cattaneo, op. cit. nota 1, pp. 70-73, figg. 21-24, in riferimento a cornici e architravi attribuiti al VII secolo.

4. Questa rilevante figura di giovane studioso è stata di recente ben indagata da Antonella Ballardini (« Da ornamento a monumento: la scultura altomedievale nella storiografia di secondo Ottocento ", Medioevo: immagine e memoria, Atti del Convegno internazionale di studi, Parma, 23-28 settembre 2008, a cura di Arturo Carlo Quintavalle, I convegni di Parma 11, Milano, Electa, 2009, pp. 109-126) e da Eugenio Russo ("Raffaele Cattaneo a centocinquant'anni dalla nascita ", Bizantinistica. Rivista di Studi Bizantini e Slavi, s. II, vol. XIII, 2011, pp. 247-269). Recentissimo è infine l'intervento di Manuela Gianandrea, " Illustrare la storia della scultura romana dei bassi tempi. Ferdinando Mazzanti e il suo corpus di disegni ", La storia dell'arte illustrata e la stampa di traduzione tra XVIII e XIX secolo, Convegno internazionale di studi, 10-11 giugno 2021, Università degli Studi di Chieti “G. D’Annunzio”, di prossima pubblicazione, con ampi riferimenti all'opera di Cattaneo.

5. E. Russo, art. cit. nota 4, p. 269.

6. Lo aveva ben sottolineato quasi cinquant'anni fa Adriano Peroni con parole incisive che vale la pena di trascrivere: « in realtà la prima storia moderna della scultura altomedievale in Italia, con risultati così avanzati da doversi dire che nessun lavoro successivo può arrogarsi di aver apportato un contributo più ampio e duraturo su questo terreno " (Adriano Peroni, " Architettura e decorazione nell'età longobarda alla luce dei ritrovamenti ", La civiltà dei Longobardi in Europa, Atti del Convegno internazionale, Roma - Cividale del Friuli, 24-28 maggio 1971, Roma, 1974, pp. 331-360, in part. p. 335). Il giudizio di Peroni è ricordato già da A. Ballardini (art. cit. nota 4, pp. 121 nota 6) e da E. Russo (art. cit. nota 4, p. 264 nota 113).

7. Ferdinando Mazzanti, "La scultura ornamentale romana nei bassi tempi ", Archivio Storico dell'Arte, ser. II, vol. II, 1896, fascicolo I-II, pp. 35-57; fascicolo III, pp. 161-187.

8. Manuela Gianandrea, La scultura altomedievale a Roma nei disegni di Ferdinando Mazzanti. La I regione ecclesiastica, con un'introduzione sull'autore e sul Fondo della Galleria Nazionale d'Arte Moderna, Corpus della scultura altomedievale, Sussidi 1, Collana diretta da Fabrizio Crivello e Francesca Romana Stasolla, Fondazione Centro Italiano di Studi sull'Alto Medioevo, Spoleto, 2021.

9. Per un'analisi comparata dell'opera e del pensiero dei due studiosi si vedano A. Ballardini (art. cit. nota 4) e soprattutto M. Gianandrea, op. cit. nota 8 e art. cit. nota 4.

10. Una efficace sintesi delle diverse posizioni assunte dagli studiosi di quei decenni è in Michelle Beghelli, Scultura altomedievale dagli scavi di Santa Maria Maggiore a Trento. Dal reperto al contesto, Bologna, BraDypUS, 2013, pp. 10-18, ma anche pp. 18-22 per i più recenti orientamenti di studi e ricerche.

11. Rudolf Kautzsch, « Die römische Schmuckkunst in Stein von 6. bis zum 10. Jahrhundert », Römisches Jahrbuch für Kunstgeschichte, vol. III, 1939, pp. 1-73; Idem, " Die langobardische Schmuckkunst in Oberitalien », ibidem, vol. V, 1941, pp. 1-48.

12. Arthur Haseloff, La scultura preromanica in Italia, Firenze, Pantheon, 1930.

13. Paolo Verzone, L'arte preromanica in Liguria ed i rilievi decorativi dei secoli "barbari", Torino, Viglongo, 1945.

14. Carlo Cecchelli, « Osservazioni sull'arte barbarica in Italia », Atti del $1^{\circ}$ Congresso Internazionale di Studi Longobardi, Spoleto, 27-30 settembre 1951, Spoleto, CISAM, 1952, pp. 137-151.

15. Géza de Francovich, « Il problema delle origini della scultura cosidetta "longobarda" ", ibidem, pp. 255-272.

16. « Ordini del giorno approvati nella seduta del 30 settembre », ibidem, pp. 23-24 
17. Mario Salmi, « Premessa », in Isa Belli Barsali, Corpus della scultura altomedievale, I, La diocesi di Lucca, Spoleto, Centro italiano di studi sull'alto medioevo, 1959, p. 5.

18. Fa eccezione il volume della diocesi di Genova la cui premessa si deve a Edoardo Arslan. L'ultimo volume introdotto da Salmi è stato quello della diocesi di Arezzo del 1977. In seguito si susseguirono Carlo Guido Mor, Michelangelo Cagiano de Azevedo, Letizia Pani Ermini, Angiola Maria Romanini, Silvia Lusuardi Siena, e ancora Letizia Pani Ermini fino al volume della diocesi di Pisa del 2011.

19. Manuela Gianandrea, "Riflessioni e qualche novità su alcune sculture altomedievali ora al Museo dell'Alto Medioevo a Roma ", Domus sapienter restaurata. Scritti di storia dell'arte per Marina Righetti, a cura di Anna Maria D'Achille, Antonio Iacobini, Pio Francesco Pistilli, Milano, Silvana Editoriale, 2021, pp. 176-183, con bibliografia precedente. Il Museo fu poi inaugurato dodici anni dopo, nel 1967.

20. Una prima messa a fuoco dello status editoriale del Corpus a quasi sessant'anni dalla sua nascita si deve a Claudia Barsanti e Alessandra Guiglia Guidobaldi, "Il Corpus della scultura altomedievale della Fondazione CISAM, Centro Italiano di Studi sull'Alto Medioevo di Spoleto: nuove prospettive ", Hortus Artium Medievalium, 24, 2018, pp. 53-59.

21. Claudia Barsanti, Roberta Flaminio, Alessandra Guiglia, La diocesi di Roma. La III regione ecclesiastica, Corpus della scultura altomedievale, vol. VII, tomo VII, Spoleto, Fondazione Centro Italiano di Studi sull'Alto Medioevo, 2015, pp. 99-361.

22. Tutti gli aspetti metodologici alla base della struttura dei Corpora sono stati affrontati e ben chiariti da Bianca Maria Felletti Maj nella eccellente « Nota preliminare » pubblicata in occasione dell'uscita dei primi tre volumi della diocesi di Roma: in Alessandra Melucco Vaccaro, La diocesi di Roma. La II regione ecclesiastica, Corpus della scultura altomedievale, vol. VII, tomo III, Spoleto, Centro Italiano di Studi sull'Alto Medioevo, 1974, pp. 1-6.

23. Silvana Casartelli Novelli, La diocesi di Torino, Corpus della scultura altomedievale, vol. VI, Spoleto, Centro Italiano di Studi sull'Alto Medioevo, 1974, nn. 108-148, pp. 184-228, tavv. LXXXIXcXXII.

24. Colette Dufour Bozzo, La diocesi di Genova, Corpus della scultura altomedievale, vol. IV, Spoleto, Centro Italiano di Studi sull'Alto Medioevo, 1966, nn. 12-42, pp. 35-72, tavv. XI-LIV.

25. Ibidem, rispettivamente n. 11, pp. 32-35, tav. X e n. 1, pp. 19-20, tav. I. Per la datazione di quest'ultimo all'età medio-bizantina si veda Alessandra Guiglia Guidobaldi « Appunti sulla scultura bizantina in Liguria ", Immagine e Ideologia. Studi in onore di Arturo Carlo Quintavalle, a cura di A. Calzona, R. Campari, M. Mussini, Milano 2007, pp. 33-46, in part. pp. 39-41.

26. Eleonora Destefanis, La diocesi di Piacenza e il monastero di Bobbio, Corpus della scultura altomedievale, vol. XVIII, Spoleto, Centro Italiano di Studi sull'Alto Medioevo, 2008, n. 9, pp. 108-121, tavv. IV-V.

27. Gaetano Panazza, Amelio Tagliaferri, La diocesi di Brescia, Corpus della scultura altomedievale, vol. III, Spoleto, Centro Italiano di Studi sull'Alto Medioevo, 1966, n. 28, pp. 44-48, tav. IX, fig. 29. Per un'aggiornata messa a punto della cronologia si veda Saverio Lomartire, "Lastra di recinzione presbiteriale ", Il futuro dei Longobardi. L'Italia e la costruzione dell'Europa di Carlo Magno, Catalogo della mostra, Brescia 2000, a cura di C. Bertelli, G.P. Brogiolo, Milano, 2000, scheda n. 265, pp. 249-250, fig. 146.

28. Ettore Napione, La diocesi di Vicenza, Corpus della scultura altomedievale, vol. XIV, Spoleto, Centro Italiano di Studi sull'Alto Medioevo, 2001, pp. 100-110, nn. 76, 160, pp. 191-192, 245-246, tavv. XXIX, LXX. Si vedano inoltre le puntualizzazioni dello stesso autore in: « Una maestranza altomedievale di lapicidi: l'officina berico-benacense », Hortus Artium Medievalium, vol. 8, 2002, pp. 325-336.

29. Amelio Tagliaferri, Le diocesi di Aquileia e Grado, Corpus della scultura altomedievale, vol. X, Spoleto, Centro Italiano di Studi sull'Alto Medioevo, 1981. 
30. Idem, ibidem, nn. 514-525, pp. 341-350, tavv. CLXXXI-CLXXXVII (nella chiesa di S. Eufemia); nn. 616-617, pp. 395-396, tav. CCXXV (da S. Giovanni Evangelista); nn. 622-623, pp. 399-402, tav. CCXXVII (nella chiesa di S. Maria delle Grazie).

31. Idem, ibidem, n. 636, pp. 409-410, tav. CCXXXII.

32. Idem, ibidem, nn. 311-330, pp. 203-216, tavv. LXXXI-XCV.

33. Per Lucca, rappresentata da un gruppo limitato di materiali, si possono indicare alcuni pilastrini reimpiegati in S. Micheletto, attribuiti all'VIII secolo, e due plutei con croci e figure animali al Museo Nazionale, forse di analoga cronologia: Isa Belli Barsali, La diocesi di Lucca, Corpus della scultura altomedievale, vol. I, Spoleto, Centro Italiano di Studi sull'Alto Medioevo, 1959, rispettivamente nn. 24-29, pp. 33-36, tavv. XII-XIV, e nn. 32-33, 34, pp. 37-39, tavv. XV-XVI.

34. Alberto Fatucchi, La diocesi di Arezzo, Corpus della scultura altomedievale, vol. IX, Spoleto, Centro Italiano di Studi sull'Alto Medioevo, 1977, rispettivamente nn. 53-54, pp. 74-76, tavv. XXXXXXIII e nn. 118, 121, pp. 132-134, 135-137, tavv. LXXIV-LXXVI, LXXIX-LXXXI.

35. Idem, ibidem, nn. 150-157, pp. 160-165, tavv. XCV-XCIX.

36. Maria Laura Testi Cristiani, La diocesi di Pisa, Corpus della scultura altomedievale, vol. XIX, Spoleto, Centro Italiano di Studi sull'Alto Medioevo, 2011, rispettivamente n. 129, pp. 217-229, tav. LVIII ("Lastra prima" di Lupeta) e n. 141, pp. 238-249, tav. LXIII ("Lastra seconda" di Lupeta).

37. Joselita Serra, La diocesi di Spoleto, Corpus della scultura altomedievale, vol. II, Spoleto, Centro Italiano di Studi sull'Alto Medioevo, 1961, n. 12, pp. 19-25, tav. V.

38. Donatella Scortecci, La diocesi di Orvieto, Corpus della scultura altomedievale, vol. XVI, Spoleto, Centro Italiano di Studi sull'Alto Medioevo, 2003, n. 10, pp. 56-63, tavv. IV-VI.

39. Gioia Bertelli, Le diocesi di Amelia, Narni, Otricoli, Corpus della scultura altomedievale, vol. XII, Spoleto, Centro Italiano di Studi sull'Alto Medioevo, 1985, n. 103, pp. 193-198, tav. XLVII.

40. Idem, ibidem, n. 73, pp. 156-160, tav. XXXII.

41. Particolarmente esemplificative sono alcune lastre di Civita Castellana, di Castel S. Elia a Nepi e di S. Pietro a Tuscania, della prima metà o della metà del IX secolo: Joselita Raspi Serra, Le diocesi dell'Alto Lazio (Bagnoregio, Bomarzo, Castro, Civita Castellana, Nepi, Orte, Sutri, Tuscania), Corpus della scultura altomedievale, vol. VIII, Spoleto, Centro Italiano di Studi sull'Alto Medioevo, 1974, rispettivamente n. 67, pp. 79-80, tav. XLIV, fig. 80; nn. 155-156, pp. 140-141, tavv. CVIII-CIX, fig. 177-178 ; nn. 384-385, pp. 272-273, tavv. CCLXIV-CCLXV, fig. 439-440.

42. Idem, ibidem, n. 43, pp. 64-67, tav. XXXI, fig. 55.

43. Idem, ibidem, nn. 180-197, pp. 154-166, tavv. CXXX-CXLIV, fig. 210-235.

44. Fabio Betti, La diocesi di Sabina, Corpus della scultura altomedievale, vol. XVII, Spoleto, Centro Italiano di Studi sull'Alto Medioevo, 2005, nn. 3-65, tavv. II-XXXIII.

45. Gioia Bertelli, Le diocesi della Puglia centro-settentrionale (Aecae, Bari, Bovino, Canosa, Egnathia, Herdonia, Lucera, Siponto, Trani, Vieste), Corpus della scultura altomedievale, vol. XV, Spoleto, Centro Italiano di Studi sull'Alto Medioevo, 2002, nn. 124-125, pp. 150-152, tav. XL.

46. Idem, ibidem, nn. 377-380, pp. 315-319, tavv. CXXI-CXXIII.

47. Mario Rotili, La diocesi di Benevento, Corpus della scultura altomedievale, vol. V, Spoleto, Centro Italiano di Studi sull'Alto Medioevo, 1966, n. 41, p. 57, tav. XIVb.

48. La I regione comprende l'area dell'Aventino con gli eccezionali complessi di S. Sabina e di S. Saba cui si aggiungono le chiese di S. Balbina e dei SS. Bonifacio e Alessio (Margherita Trinci Cecchelli, La diocesi di Roma. La I regione ecclesiastica, Corpus della scultura altomedievale, vol. VII, tomo IV, Spoleto, Centro Italiano di Studi sull'Alto Medioevo, 1976). La II regione occupa una vasta area del centro monumentale più antico, dal Velabro al Celio fino al complesso della Basilica Lateranense e contiene alcune tra le chiese più importanti per il periodo altomedievale come S. Giorgio al Velabro, S. Maria in Cosmedin, S. Stefano Rotondo, S. Maria in Domnica e SS. Quattro Coronati (Alessandra Melucco Vaccaro, La diocesi di Roma. La II regione ecclesiastica, Corpus della scultura altomedievale, vol. VII, tomo III, Spoleto, Centro Italiano di Studi sull'Alto Medioevo, 1974). La III regione si estende dalle Mura Aureliane, presso la via Tiburtina, 
all'Esquilino e alla valle del Colosseo fino ai confini del Celio e include le chiese di S. Croce in Gerusalemme, S. Bibiana, S. Martino ai Monti e S. Clemente (C. Barsanti, R. Flaminio, A. Guiglia, op. cit. nota 21). La IV regione, infine, si concentra nel territorio fittamente abitato posto tra i colli Quirinale, Viminale e in parte Esquilino, compresa l'antica Subura, con le chiese di S. Maria Maggiore, S. Pudenziana, S. Prassede e S. Agata dei Goti, per citare le più significative (Letizia Pani Ermini, La diocesi di Roma. La IV regione ecclesiastica, Corpus della scultura altomedievale, vol. VII, tomo I, Spoleto, Centro Italiano di Studi sull'Alto Medioevo, 1974).

49. Umberto Broccoli, La diocesi di Roma. Il Suburbio, I, Corpus della scultura altomedievale, vol. VII, tomo V, Spoleto, Centro Italiano di Studi sull'Alto Medioevo, 1981.

50. Alessandra Melucco Vaccaro, Lidia Paroli, La diocesi di Roma. Il Museo dell'Alto Medioevo, Corpus della scultura altomedievale, vol. VII, tomo VI, Spoleto, Centro Italiano di Studi sull'Alto Medioevo, 1995.

51. Letizia Pani Ermini, La diocesi di Roma. La raccolta dei Fori Imperiali, Corpus della scultura altomedievale, vol. VII, tomo II, Spoleto, Centro Italiano di Studi sull'Alto Medioevo, 1974.

52. Maria Grazia Branchetti, "Per un catalogo della scultura altomedievale a Roma, II. I reperti del Museo di Roma ", Studi Romani, vol. 20, 1972, pp. 373-382; Anna Maria Ramieri, « Frammenti inediti di sarcofagi e rilievi altomedievali da "via del Mare" in Roma ", Rivista di Archeologia Cristiana, vol. LXXVIII, 2002, pp. 301-323; Fabio Betti, " Sculture carolingie del Lapidario del Museo di Roma. Materiale inedito e contesti di provenienza ", Bollettino dei Musei Comunali di Roma, n.s. vol. 17, 2003, pp. 142-161 ; Donatella Germanò, Claudia Ornaghi, « Per la conoscenza della collezione di scultura altomedievale del Museo di Roma ", Studi Romani, n.s. I,2, 2019, pp. 39-98.

53. M. Gianandrea, op. cit. nota 8.

54. Per le complesse e assai articolate vicende dell'acquisizione del Fondo si veda ora Idem, ibidem.

55. Lorenza de Maria, Francesca Fei, Angela Toro, Disegni e spolveri. I marmi medioevali del Lazio nel "Fondo Mazzanti", a cura di Francesca Fei, Roma, Palombi Editori, 2002.

56. Claudia Barsanti, Alessandra Guiglia Guidobaldi, « Gli elementi della recinzione liturgica ed altri frammenti minori nell'ambito della produzione scultorea protobizantina ", in Federico Guidobaldi, Claudia Barsanti, Alessandra Guiglia Guidobaldi, San Clemente. La scultura del VI secolo, San Clemente Miscellany IV,2, Roma, apud S. Clementem, 1992, pp. 67-270, in part. pp. 265-270, figg. 400-408: Nota aggiuntiva.

57. Fabio Betti, «L'arredo liturgico della basilica di S. Sabina al tempo di papa Eugenio II: dalla scoperta ai restauri storici $(1894,1918,1936)$ », Arte Medievale, IV serie, anno VII, 2017, pp. 31-52.

58. Alessandra Guiglia, "Aggiornamenti sulle sculture altomedievali del complesso di San Gregorio al Celio ", Inedita medioevalia. Scritti in onore di Francesco Aceto, a cura di Francesco Caglioti e Vinni Lucherini, Roma, Viella, 2019, pp. 237-246, in part. fig. 5; M. Gianandrea, art. cit. nota 19.

\section{RIASSUNTI}

L'articolo intende presentare il contributo fondamentale offerto dal Corpus della scultura altomedievale, edito fin dal 1959 dal Centro Italiano di Studi sull'Alto Medioevo di Spoleto, poi Fondazione CISAM, per la conoscenza del patrimonio scultoreo di quei secoli in Italia. Dopo una 
breve introduzione sulle figure di studiosi che, a partire dalla fine dell'Ottocento, hanno aperto la via agli studi moderni sulla scultura altomedievale, vengono ripercorse le vicende che hanno visto la nascita del progetto Corpus e la pubblicazione dei 25 volumi. Di essi vengono evidenziati i caratteri principali, la metodologia espositiva e infine i mutamenti che si sono susseguiti nel corso dei 56 anni trascorsi dal primo all'ultimo volume uscito. Vengono poi messe sinteticamente a fuoco la varietà e la ricchezza dei materiali, nonché le peculiarità che contraddistinguono le diverse aree storico-culturali e artistiche del territorio italiano. A conclusione vengono esposti i due progetti in corso sulle sculture del Lapidario del Museo di Roma a Palazzo Braschi e sul Fondo dei disegni di Ferdinando Mazzanti alla Galleria Nazionale d'Arte Moderna di Roma.

L'objectif de cet article est de présenter la contribution fondamentale apportée par le Corpus de la sculpture du haut Moyen Âge, publié depuis 1959 par le Centro Italiano di Studi sull'Alto Medioevo de Spoleto, devenu ensuite la Fondazione CISAM, pour la connaissance du patrimoine sculptural de ces siècles en Italie. Après une brève introduction sur les chercheurs qui, depuis la fin du XIXe siècle, ont ouvert la voie aux études modernes sur la sculpture du haut Moyen Âge, les événements qui ont conduit à la naissance du projet de Corpus et à la publication des 25 volumes sont retracés. Les principales caractéristiques des 25 volumes, la méthode de présentation et les changements intervenus au cours des 56 années écoulées depuis la publication du premier volume sont mis en évidence. La variété et la richesse des matériaux sont ensuite brièvement évoquées, ainsi que les particularités qui distinguent les différentes zones historiques, culturelles et artistiques de l'Italie. Le livre se termine par une présentation des deux projets en cours sur les sculptures du Lapidarium du Museo di Roma au Palazzo Braschi et sur la collection de dessins de Ferdinando Mazzanti à la Galleria Nazionale d'Arte Moderna de Rome.

\section{INDICE}

Parole chiave : scultura, alto medioevo, Italia, Roma, Corpus, Raffaele Cattaneo, Ferdinando Mazzanti, disegni di sculture

Mots-clés : sculpture, haut Moyen Âge, Italie, Rome, Corpus, Raffaele Cattaneo, Ferdinando Mazzanti, dessins de sculptures

\section{AUTORE}

\section{ALESSANDRA GUIGLIA}

Alessandra Guiglia, ancien professeur titulaire d'histoire de l'art byzantin à l'université de la Sapienza de Rome, a mené ses premières recherches sur l'architecture médiévale en Lombardie et sur la peinture au Moyen Âge romain. Elle a ensuite concentré ses études sur la culture artistique de la Méditerranée orientale, en particulier sur la décoration des sols et la sculpture architecturale et liturgique de production constantinopolitaine, ainsi que sur la décoration murale en mosaïque byzantine. De 1999 à 2014, elle a coordonné, avec Claudia Barsanti, le projet de recherche sur le mobilier en marbre de Sainte-Sophie à Constantinople et sur les sculptures de l'Ayasofya Müzesi. Plus récemment, elle s'est intéressée à la sculpture romaine du haut Moyen Âge dans le cadre des publications du Corpus de la sculpture du haut Moyen Âge édité par la Fondazione Centro Italiano di Studi sull'Alto Medioevo de Spoleto.

Alessandra Guiglia, già professore ordinario di Storia dell'arte bizantina alla Sapienza Università di Roma, ha condotto le sue prime ricerche sull'architettura medievale lombarda e sulla pittura del medioevo romano. In seguito ha orientato i suoi studi verso la cultura artistica del Mediterraneo orientale, in particolare la decorazione pavimentale e la scultura architettonica e 
liturgica di produzione costantinopolitana, ma anche la decorazione musiva parietale di ambito bizantino. Dal 1999 al 2014 ha coordinato, insieme a Claudia Barsanti, il progetto di ricerca sull'arredo marmoreo di Santa Sofia a Costantinopoli e sulle sculture dell'Ayasofya Müzesi. Più recentemente ha rivolto l'attenzione alla scultura altomedievale romana nell'ambito delle pubblicazioni del Corpus della scultura altomedievale edito dalla Fondazione Centro Italiano di Studi sull'Alto Medioevo di Spoleto. 